\section{Universidades y promoción de la salud: ¿cómo alcanzar el punto de encuentro?}

\author{
Mónica Muñoz \\ y Baltica Cabieses ${ }^{1}$
}

Forma de citar: Muñoz M, Cabieses B. Universidades y promoción de la salud: ¿cómo alcanzar el punto de encuentro? Rev Panam Salud Pública. 2008;24(2):139-46.

Palabras clave: promoción de la salud, educación superior, calidad de vida, desarrollo humano, Chile.

\footnotetext{
1 Programa UC Saludable. Pontificia Universidad Católica de Chile. La correspondencia se debe enviar a Mónica Muñoz. Directora Programa UC Saludable. Directora Extensión. Pontificia Universidad Católica de Chile. Vicuña Mackenna 4860. Macul, Santiago 6904411, Chile. T.: 56-2-3545303. F.: 56-2-3547025. Correo electrónico: mmunozse@uc.cl
}

Las universidades desempeñan un papel protagónico en la sociedad donde están insertas. Orientadas al liderazgo y al desarrollo del conocimiento, son escenario de formación técnica y profesional de los miembros de la comunidad $\mathrm{y}$, a través de la educación, la investigación y la difusión del saber, orientan y apoyan los cambios en diversos espacios nacionales e internacionales. Toda universidad cumple su misión social al penetrar en la cultura en que está inmersa, mediante el avance constante del saber en los temas de mayor relevancia y necesidad para cada país. De esta forma, las instituciones de educación superior buscan comprender los fenómenos y aportar soluciones que enriquezcan y orienten el futuro nacional, regional y mundial. En otras palabras, la existencia misma de una universidad implica su participación en la cultura y en los procesos relevantes de la nación a la que pertenece.

La misión social de las universidades se vincula estrechamente con su tarea formativa; así entonces, tomando en cuenta el carácter nacional y de servicio señalado, y tal como lo destacara en 2000 el doctor Pedro Pablo Rosso, rector de la Pontificia Universidad Católica de Chile (1), las universidades deben aspirar al protagonismo en el cambio cultural mediante la educación de personas más cultas, abiertas, emprendedoras e íntegras, comprometidas con el desarrollo progresivo de la sociedad. Sin embargo, este cambio cultural exige desafíos enormes para las universidades, pues no sólo demanda una formación de excelencia en cada disciplina, sino que requiere el fortalecimiento de conductas y actitudes transversales que adoptará cada profesional, quien construirá y tomará decisiones al interior de la sociedad. Surge aquí una segunda condición para la existencia de una universidad, y que se refiere al desarrollo permanente de las capacidades de los miembros de la universidad.

El concepto de promoción de la salud ha quedado sólidamente implementado en diversos ámbitos nacionales, tales como las escuelas, las comunas, las municipalidades, los ministerios, etc., gracias a los lineamientos desarrollados tanto en América Latina como en el resto del mundo. Ejemplo de estos últimos son, en orden cronológico, la Declaración de Alma Ata 1978 (2); la Carta de Ottawa 1986 (3, 4); la Conferencia de Adelaida, Australia 1988 (5); la Declaración de Promoción de Salud en América Latina 1992 (6); la Conferencia de Trinidad y Tobago 1993 (6, 7); el Seminario Internacional de Francia 1994 (6); la Declaración de Yakarta 1997 (5, 
8, 9); la Resolución de la Asamblea Mundial de la Salud 1998 (6); la Declaración de Medellín, Colombia 1999 (10); la Declaración Ministerial de México 2000 (5); el Foro Nacional de El Salvador 2002 (6); la Resolución de Copán Ruinas, Honduras 2001 (6); el Informe Final de Managua, Nicaragua 2001 (6); el Foro de Chile 2002 (6, 11); la Carta de Sundsvall 2004 (5); la Conferencia de Bangkok 2005 (5) y el Foro de Río de Janeiro 2006 (12).

$\mathrm{Si}$ bien las universidades no asumieron este desafío de manera inmediata, tanto la Organización Panamericana de la Salud (OPS) como la Organización Mundial de la Salud (OMS) (13) han reconocido su relevancia al señalar que estas instituciones promueven y apoyan la colaboración entre los sectores del gobierno, los sectores privado y público y los socios no gubernamentales de cada país, así como también en el plano internacional

Una universidad promotora de la salud es aquella que incorpora el fomento de la salud a su proyecto, con el fin de propiciar el desarrollo humano y mejorar la calidad de vida de quienes allí estudian o trabajan, influyendo así en entornos laborales y sociales. De esta manera promueve conocimientos, habilidades y destrezas para el propio cuidado y para la implantación de estilos de vida saludables en la comunidad universitaria (14). ${ }^{2}$

En este trabajo se busca dar a conocer un análisis reflexivo en torno a la necesaria asociación que debe existir entre el ámbito universitario y la promoción de la salud, con el fin de motivar y proporcionar líneas de acción a otras instituciones de educación superior, tanto chilenas como del resto de Latinoamérica. Dicho análisis surge, por una parte, de la experiencia que durante varios años ha desarrollado la Pontificia Universidad Católica de Chile, a través del Programa UC Saludable y en alianza colaborativa con la OPS y, por la otra, de la sistemática revisión de la literatura acerca del tema.

\section{HACIA UN ENCUENTRO ENTRE UNIVERSIDAD Y PROMOCIÓN DE LA SALUD}

La comunidad universitaria está formada por un cuerpo docente, uno estudiantil y otro administrativo. Cada uno de estos grupos desempeña un papel fundamental en el proceso de enseñanzaaprendizaje y tiene la responsabilidad de liderar, orientar y/o apoyar las estrategias educativas que allí se proponen. Al mismo tiempo, la interacción constante y dinámica entre estos grupos promueve

\footnotetext{
2 Programa UC Saludable. Informe diagnóstico estilos de vida de estudiantes UC. Santiago: Pontificia Universidad Católica de Chile; 2001.[Documento no publicado]
}

una cultura local, donde se enmarcan y delimitan las formas de ser y de comportarse. Cada académico, estudiante y administrativo de la universidad construye esta cultura local, misma que se da a conocer no tanto a través del discurso de aula, sino mediante las conductas personales. Es sabido que los jóvenes no anhelan escuchar discursos brillantes sino comprobar con hechos concretos lo que sus formadores afirman ser $\mathrm{y}$, si bien su estancia en la universidad es temporal, se encuentran en una etapa del ciclo vital clave para la adopción de estilos de vida que luego practicarán en el ámbito familiar, social y laboral.

Lo anterior coincide con el concepto de promoción de la salud, definido en la Carta de Ottawa de 1986: "la salud es construida y vivida por las personas en su ambiente cotidiano, donde ellas trabajan, aprenden, juegan y aman" (3). En este sentido, si una universidad asume el desafío de ser promotora de la salud, debe entonces conocer, comprender, apoyar y fortalecer los estilos de vida saludables para cada uno de los grupos de esa comunidad.

Sin embargo, la modificación de conductas y actitudes que generen una cultura saludable no depende únicamente de las personas, sino también de la estructura, políticas y decisiones estratégicas que se adopten desde los cargos directivos de las universidades. Lo anterior se sustenta en el concepto de "ciudades saludables", que da prioridad a iniciativas de promoción de la salud dirigidas a la universidad como entorno, mediante la "construcción de ambientes físicos, psíquicos y sociales que influyan en mejorar la calidad de vida de la comunidad universitaria" (14). El entorno es el estímulo para que las personas emprendan cambios positivos en su conducta; por consiguiente, a los encargados de tomar decisiones al interior de la universidad les corresponde la responsabilidad de proveer las condiciones idóneas. Como lo señaló la OPS en 2002:

La promoción de la salud es un proceso, no una solución fácil. Su propósito es lograr un resultado concreto en un plazo largo, pero con efectos a mediano y corto plazos. Los resultados concretos varían, comprenden la participación de los ciudadanos y la comunidad y contribuyen a mejorar la calidad de vida (15).

Así entonces, como protagonista del desarrollo de un país y de una región, la universidad tiene un papel clave en el establecimiento de conocimientos, conductas y actitudes que promuevan una cultura más saludable y equitativa. Para ello, y de acuerdo con la creciente necesidad de los países de promover estilos de vida saludables en los escenarios nacionales y regionales, el entorno universitario ha cobrado cada vez mayor relevancia y lide- 
razgo. A pesar de que no se conocen con claridad todas las dimensiones del papel de las instituciones de educación superior como agentes promotores de la salud y del bienestar, existen interesantes experiencias en diversos países latinoamericanos que, a través del trabajo sistemático, han permitido desarrollar un cuerpo sólido de conocimiento en torno a este tema. Toda universidad preocupada en promover la salud puede encauzar sus esfuerzos a cuatro aspectos centrales que a continuación se abordan.

\section{A. Responder a la problemática de educación y salud del país y la región}

La salud de cada país y región varía de acuerdo con su realidad geográfica, económica, social y cultural; sin embargo, existe una marcada tendencia regional al aumento de las enfermedades crónicas no transmisibles, que dependen directamente de los estilos de vida individuales (16). Como lo señaló la presidenta de la República de Chile, Michelle Bachelet J.:

Tanto los aspectos medioambientales como los socioeconómicos, el avance y la masificación del consumo, la apertura internacional y el desarrollo tecnológico, han creado condiciones para nuevos hábitos de vida no saludables, entre ellos el sedentarismo, la obesidad y el tabaquismo (14).

Desde la perspectiva local, Chile ha tenido cambios muy rápidos en su perfil epidemiológico. De una situación pre transicional, con predominio de enfermedades infecciosas y materno-infantiles en la década de 1960, pasó rápidamente a una situación post transicional con predominio de enfermedades crónicas no transmisibles, accidentes y problemas de salud mental a fines de los ochenta, que se mantienen vigentes en la actualidad (17).

En cuanto a la educación, si bien desde hace algún tiempo se ha realizado trabajo de promoción de la salud en el escenario educativo escolar, en el ámbito universitario este proceso ha sido más lento y menos sólido, lo cual se ha combinado con la dramática transformación que sufre la educación superior desde hace algunas décadas. En Chile por ejemplo, de una población menor a 100 mil estudiantes en los años ochenta, se pasó a más de 700 mil en los últimos años. Como se señala en la Guía para universidades promotoras de la salud y otras instituciones de educación superior (14), esta población estudiantil, en conjunto con los académicos y el personal administrativo, además de sus familias, constituye un universo significativo para el crecimiento y desarrollo de un país. Luego entonces, es necesario impulsar la promoción de la salud como objetivo transversal de las universidades, tanto para su implementación local como para su desarrollo nacional e internacional.

Los principales retos de los países están fundamentados, por lo tanto, en: (a) las profundas transformaciones sociales de las últimas décadas; (b) la tendencia mundial a una mirada convergente de los retos que el mundo enfrenta, así como a las estrategias más amplias para encararlos; (c) la convicción de que pueden lograrse grandes avances mediante un sólido liderazgo nacional e internacional en la gestión, distribución y articulación de los recursos disponibles, y (d) un enfoque intersectorial que involucre, entre otros, al sector productivo, a la salud y a la educación, incorporando a las universidades. En este sentido, la promoción de la salud ha surgido como una estrategia importante para lograr equidad, democracia y justicia social. Constituye un proceso político y social global que abarca no sólo las acciones dirigidas específicamente a fortalecer las habilidades y capacidades de los individuos, sino también aquellas destinadas a modificar condiciones adversas sociales, ambientales y económicas del país, con el fin de mitigar su impacto en la salud pública e individual.

La promoción de la salud es un proceso que permite incrementar el control sobre los determinantes de la salud (3), a través del cual se pueden abordar complejos problemas sanitarios, sociales y económicos, y que ofrece un marco valioso para organizar la acción social y política con el fin de mejorar la salud y las condiciones de vida. La promoción de la salud constituye, por ende, un enfoque técnico, político, social y académico para trabajar con distintos sectores y mejorar la calidad de vida de las personas (13).

Las universidades tienen un papel protagónico en la promoción de la salud como eje central del desarrollo de la educación y de la salud de un país y de una región, puesto que son capaces de asumir la responsabilidad social que implica ser una institución de educación superior, en búsqueda constante de una mayor interconexión para mejorar el intercambio de conocimientos y las transferencias de tecnología, e impulsando el desarrollo permanente del conocimiento como estrategia fundamental para reordenar las prácticas de formación, atención, administración, formulación de políticas y participación social en promoción de la salud y, por último, encabezando y facilitando acciones intersectoriales oficiales y regulares con el sector de la salud y de la educación. Todos estos desafíos, así como la necesidad de las universidades de asumirlos, han sido detallados por la OPS/OMS, de la siguiente manera:

Las universidades pueden ser aliados importantes en la realización de la investigación y 
evaluación de la promoción de la salud. . . En diversos países hay fuentes de datos que, si se analizan, interpretan y presentan adecuadamente, podrían atender muchas necesidades de promoción de la salud, como aumentar las pruebas de su efectividad, compilar las enseñanzas extraídas y la documentación de las experiencias (13).

Hoy en día muchos consideran la promoción de la salud como la nueva salud pública debido, en primer lugar, a que su enfoque no se limita a las enfermedades y, en segundo lugar, a que ofrece herramientas para enfrentar el cambio del perfil sociodemográfico y epidemiológico de un país, a la vez que permitirá promover modos de vida y espacios sociales sanos y prevenir tanto las enfermedades infecciosas como aquellas no transmisibles. El entorno educativo -como la universidad, por ejemplo- es el más propicio para generar una cultura nacional y regional de la salud, en tanto que da lugar a cambios saludables en el comportamiento y permite trabajar con un grupo sensible de la población; además, encabeza procesos de desarrollo del conocimiento relevantes para la salud nacional e internacional y permite articular estrategias con grupos sociales diversos.

\section{B. Generar e intercambiar conocimiento en torno a la promoción de la salud}

Resulta interesante retomar los grandes cambios que las universidades han experimentado durante las últimas décadas, como resultado de las crecientes exigencias en educación, investigación y permanente diálogo con la sociedad. Algunas instituciones universitarias han experimentado, por ejemplo, modificaciones en su capacidad académica - cantidad de estudiantes y profesores, número de programas de estudio, productividad académica, capacidad de investigación- y la consecuente transformación que las ha hecho pasar de ser "universidades docentes", centradas en la formación de profesionales, a "universidades de investigación" (18). Este tipo de universidades ha requerido de académicos de tiempo completo, expertos en las disciplinas que enseñan y capaces de generar investigación relevante. Para ello, la formación de postgrado se transforma en un elemento crucial para alcanzar el objetivo de generar e intercambiar conocimiento en torno a la promoción de la salud y es, al mismo tiempo, uno de los principales desafíos de las universidades contemporáneas.

En términos generales, estas instituciones se organizan en líneas estratégicas enfocadas en el proyecto educativo, la generación de conocimiento, los vínculos con la sociedad y la gestión administrativa. En apoyo a la idea anterior, se puede afirmar que el vínculo con la sociedad se refleja en el diálogo permanente con el gobierno, la realización de estudios sobre problemáticas relevantes para la nación y la propuesta de alternativas de solución.

En el ámbito social, la colaboración de las instituciones de educación superior debería enfocarse a la promoción de los individuos a través de la educación y la capacitación laboral, el fomento de la salud y la aplicación de políticas públicas. En el campo de la economía, el esfuerzo tendría que orientarse al desarrollo y aplicación de políticas eficaces, a la creación y fortalecimiento de empresas, al aumento de la productividad, y al desarrollo de nuevos y mejores productos. En el plano cultural, se debe trabajar para construir una sociedad más humana, creando puentes hacia un futuro más próspero, justo y solidario. En el campo de la salud, las universidades tendrían que desarrollar modelos de intervención efectivos en áreas de considerable interés y relevancia como son el ambiente físico y psicosocial, la alimentación, la actividad física y las adicciones que, en el futuro, podrían ser aplicados en una escala nacional. La Pontificia Universidad Católica de Chile por ejemplo, ha puesto en marcha desde el año 2000 el Programa UC Saludable, que intenta promover estilos de vida sanos en esa comunidad, modificando hábitos y conductas que generan riesgos de salud. Este proyecto es uno de los primeros de su tipo realizado en el ámbito de la educación superior en Chile y ha permitido avanzar en el conocimiento en torno a la promoción de la salud en el escenario universitario. Sus principales logros se orientan al fomento de una vida saludable en áreas seleccionadas como prioritarias, a saber: actividad física, alimentación, ambientes libres de humo de tabaco, sexualidad responsable y seguridad personal. Tras siete años de experiencia, se puede argumentar que el desarrollo de la promoción de la salud en el escenario universitario demanda expertos en el área que sean capaces de proponer, poner en marcha, evaluar y sintetizar el trabajo y las alianzas estratégicas acumuladas, en los planos nacional e internacional.

Como profesionales con formación de postgrado, expertos en investigación y en disciplinas directa e indirectamente relacionadas con la promoción de estilos de vida saludables, los académicos universitarios tienen grandes desafíos con respecto al desarrollo del conocimiento en promoción de la salud. El personal administrativo por su parte, es un pilar que sustenta los procesos internos de cambio hacia la promoción de la salud y que reflejan la realidad de la población adulta de cada país. Y por último los estudiantes, que cada día son más exigentes en lo tocante a su formación profesional, son 
parte activa en la realización de actividades que favorezcan su salud, capaces de liderar procesos de cambio en sus vidas y al interior de la universidad. Los miembros de la comunidad universitaria, tanto jóvenes como adultos, requieren de conocimientos actualizados, de habilidades concretas y de motivación permanente para cuidar su salud de manera efectiva y duradera.

\section{Orientar y apoyar la educación para el autocuidado y los estilos de vida saludables}

Resulta imposible abordar el tema de la promoción de la salud sin hablar de la relevancia que cobra la educación para el cuidado de la propia salud y los estilos de vida saludables. En este sentido, las instituciones de educación superior son las grandes responsables de la generación de modelos, teorías y aplicaciones prácticas del saber que permiten implementar, en la vida cotidiana por ejemplo, el concepto dinámico y versátil de promoción de la salud. Si bien la visión de los puntos anteriores ha sido comunitaria al responder a las necesidades de un país en los temas de educación y salud, a continuación se incluirá la perspectiva del individuo, a través de las capacidades y necesidades en el proceso de aprendizaje en torno al cuidado de sí mismo.

La educación en promoción de la salud incluye el concepto medular de autocuidado, que proviene del modelo de Dorothea Orem y cuya filosofía sostiene que todo ser humano es capaz de cuidar y aprender a cuidar de sí mismo. Para ello, cada persona requiere de conocimientos pertinentes, de habilidades motoras para la acción y de motivación para realizar ese cuidado. De esta manera, el autocuidado se ve reflejado en conductas que existen en situaciones concretas de la vida, dirigidas por las personas hacia sí mismas o hacia el entorno, para regular los factores que afectan su propio desarrollo y funcionamiento en beneficio de su vida, salud y bienestar (19). El autocuidado se entrelaza con la promoción de la salud al facultar a los individuos y las comunidades para que aborden sus propios temas y al utilizar la energía y creatividad de cada persona mediante la acción. Al mismo tiempo, y dentro del contexto de su promoción, la salud se considera no como un estado abstracto sino como un medio para llegar a un fin, es decir, como un recurso que permite a los individuos llevar una vida personal, social y económicamente productiva. La salud es, entonces, un recurso para la vida diaria, y no el objetivo de la vida. Se trata de un concepto positivo que pone el acento en los recursos sociales y personales, así como en las aptitudes físicas (19).

La promoción de la salud y el autocuidado se relacionan también íntimamente con el concepto de educación para la salud (health education), que comprende oportunidades de aprendizaje, incluido un mejor conocimiento de la población en relación con la salud y el desarrollo de habilidades personales que conduzcan a la salud personal y de la comunidad. La educación para la salud, en consecuencia, aborda no sólo la transmisión de información, sino también el fomento de la motivación, las habilidades personales y la autoestima, necesarias para adoptar medidas destinadas a mejorar la salud. Como lo plantea la OMS:

La educación para la salud incluye no sólo la información relativa a las condiciones sociales, económicas y ambientales subyacentes que influyen en la salud, sino también la que se refiere a los factores de riesgo y comportamientos de riesgo, además del uso del sistema de asistencia sanitaria. Es decir, la educación para la salud supone comunicación de información y desarrollo de habilidades personales que demuestren la viabilidad política y las posibilidades organizativas de diversas formas de actuación dirigidas a lograr cambios sociales, económicos y ambientales que favorezcan la salud (19).

El último aspecto interesante que cabe considerar en materia de educación para el autocuidado y los estilos de vida saludables, se relaciona con el aprendizaje constante de los determinantes de la salud, pues la actuación en este ámbito tiene un gran potencial para reducir los efectos negativos de las enfermedades y promover la salud de la población. Rodríguez (20) explica este fenómeno de la siguiente manera:

Los comportamientos saludables se establecen, cambian o extinguen en el repertorio de la persona, en primer lugar por condicionamiento y en segundo lugar por los modelos a través del aprendizaje vicario, que es una de las maneras más importantes de aprendizaje del ser humano. En tercer lugar, los factores cognitivos tienen un papel relevante en la adquisición y mantenimiento de la conducta de promoción de la salud. Cada uno de nosotros valora su estado de salud y toma decisiones a favor de ella conforme a sus creencias, a su contexto sociocultural y a su conocimiento.

Los factores determinantes de la salud pueden dividirse en las siguientes categorías: comportamiento y estilo de vida personal; influencias dentro de las comunidades que pueden reforzar la salud o dañarla; las condiciones de vida y de trabajo y el acceso a los servicios sanitarios; y las condicio- 
nes generales de tipo socioeconómico, cultural y ambiental (21).

Los factores determinantes de la salud relacionados con el estilo de vida poseen múltiples dimensiones y están ligados a varios problemas importantes para la salud. Pueden ser específicos de una etapa del ciclo vital, pero también pueden estar muy vinculados con aspectos culturales y a factores socioeconómicos que, por sí mismos, constituyen un motivo importante de variaciones en la salud (22). A continuación se mencionan diez reconocidos determinantes de la salud asociados a los estilos de vida: consumo de tabaco; nutrición insuficiente o excesiva (hábitos alimentarios); ejercicio aeróbico insuficiente; consumo excesivo de alcohol; ausencia de inmunización contra agentes microbianos; exposición a toxinas y venenos; armas de fuego; comportamientos sexuales de riesgo; traumatismos por vehículos automotores, y consumo de drogas ilegales $(23,24)$.

$\mathrm{Al}$ hacer referencia a los determinantes socioeconómicos de la salud, resulta inevitable hablar de la desigualdad en materia de salud, que disminuye la capacidad de muchos individuos para lograr su plena realización. En la promoción de la salud surgen diversas acciones orientadas a mejorar esa realidad, como son incrementar el nivel general de salud acercándolo al de los más favorecidos, garantizar la plena satisfacción de las necesidades sanitarias de los más desfavorecidos, y ayudar a mejorar más rápidamente la salud de las personas de los países y regiones en mayor desventaja.

Finalmente, respecto del medio ambiente, la presencia de peligros naturales o provocados por el hombre es una fuente de enfermedades relacionadas con el entorno, que podrían considerarse como la indicación visible y clínica de condiciones ambientales inadecuadas. Los principales campos de acción son la calidad del aire en el exterior y el interior, el ruido, las condiciones ambientales de la vivienda, la contaminación del agua, los campos electromagnéticos y las radiaciones y las exposiciones a agentes químicos. La repercusión de estos factores se manifiesta en ciertos problemas como los de audición, insomnio, estrés conducente a la hipertensión y otros padecimientos circulatorios, cánceres de piel y de otro tipo, asma y malformaciones congénitas (21).

\section{Ser un agente de cambio modelo para la promoción de la salud}

Para ser un agente de cambio es necesaria la movilización hacia la acción, es decir, la implementación de actividades concretas, articuladas entre sí y fundamentadas en un sólido marco conceptual, político, organizacional y de proceso. En otras palabras, la única manera de asumir la responsabilidad de ser una universidad modelo en promoción de la salud, es a través de la realización permanente y duradera de estrategias a favor de estilos de vida saludables en cada una de las personas y grupos que conforman la universidad, evaluados y perfeccionados de manera sistemática.

Ser modelo de cambio implica empoderamiento (empowerment for health). En la promoción de la salud, el empoderamiento es un proceso mediante el cual las personas adquieren un mayor control sobre las decisiones y acciones que afectan su salud. El empoderamiento para la salud es un proceso social, cultural, psicológico y/o político complejo, a través del cual tanto los individuos como los grupos sociales son capaces de expresar sus necesidades, plantear sus preocupaciones, diseñar estrategias de participación en la toma de decisiones y llevar a cabo acciones. De esta manera, las personas perciben una relación más estrecha entre sus metas y el modo de alcanzarlas y una correspondencia entre sus esfuerzos y los resultados que obtienen. Además de las acciones dirigidas a fortalecer las habilidades básicas para la vida y las capacidades de los individuos, la promoción de la salud incluye las acciones para influir en las condiciones sociales y económicas y en los entornos físicos que influyen sobre la salud. En este sentido, la promoción de la salud va dirigida a crear las mejores condiciones posibles a fin de que exista una relación entre los esfuerzos de los individuos y los resultados en salud que obtienen (19).

Las instituciones de educación superior deben, entonces, empoderarse en su responsabilidad como promotoras de la salud tanto en el escenario interno como en el nacional y regional, comenzando por el apoyo cotidiano de cada uno de sus miembros y la generación de entornos que promuevan estilos de vida cada vez más saludables, y obteniendo en forma paralela el apoyo de los directivos y la incorporación de la promoción de la salud a los objetivos estratégicos de la institución. Esto se apoya en lo publicado en la Carta de Ottawa para la Promoción de la Salud (3) y en la Declaración de Yakarta (25) donde, como áreas de acción prioritarias, se señalan el establecimiento de políticas saludables, la creación de entornos que apoyen la salud, el fortalecimiento de la acción comunitaria para la salud, la promoción de la responsabilidad social para la salud y el incremento de la capacidad de la comunidad y empoderamiento de los individuos en el cuidado de su propia salud. Una comunidad universitaria empoderada para su salud es aquella cuyos individuos y organizaciones aplican sus habilidades y recursos en esfuerzos colectivos destinados a abordar las prioridades sanitarias y a satisfacer sus necesidades sanitarias respectivas. Mediante esta participación, los individuos y las organizaciones de una comunidad desarrollan su capacidad para ofrecer apoyo social en materia de salud, 
enfrentar los conflictos dentro de la comunidad, y adquirir una mayor influencia y control sobre los determinantes de la salud comunitaria $(3,19,26)$.

Ser líder en el proceso de cambio implica también generar, probar y educar respecto de modelos de gestión de cambio de actitudes y conductas, apoyados en las ciencias administrativa, psicológica y sociológica, entre otras. Modelos como el de creencias en salud, el modelo social ecológico, la teoría de acción razonada (o planificada) o la teoría de acción social, por mencionar las más referidas $(20,27)$, son paradigmas de gran ayuda para observar, comprender, analizar y evaluar estrategias en torno a la promoción de la salud y que no deben dejarse de lado a la hora de iniciar actividades que promuevan estilos de vida saludables, puesto que sustentan las intervenciones, dan un marco de acción y clarifican los pasos a seguir de manera sistemática (28). Las universidades son importantes agentes de investigación en disciplinas que abordan las temáticas de hábitos, toma de decisiones y cambio de conductas en salud, y pueden facilitar significativos procesos individuales y grupales en la promoción de la salud. De esta manera, pueden convertirse en agentes de cambio modelo para la promoción de la salud y, por lo tanto, en dignos referentes para otros escenarios educativos, laborales y comunitarios en general.

Por último, y tal como se ha señalado anteriormente, la comunidad universitaria está formada por académicos, administrativos $\mathrm{y}$, en mayor proporción, por estudiantes, cabe decir por jóvenes que podrían inducir cambios de conducta en sus familias y que, en alguna medida, serán un modelo en los ámbitos laborales donde lleguen a insertarse. De esta manera, el desafío especial de la universidad está en reconocer que los jóvenes de hoy serán los adultos del mañana que tendrán la responsabilidad de enmarcar en sus familias, entornos laborales y sociales, la promoción de estilos de vida saludables.

\section{CONSIDERACIONES FINALES}

Las universidades pueden ser promotoras de la salud si se lo proponen; tienen el potencial para influir positivamente en la vida y condición de salud de sus miembros, protegiéndolos y promoviendo su bienestar y pueden liderar y apoyar procesos de cambio en la comunidad externa, a través de sus políticas y prácticas. Además, al ser organizaciones extensas y complejas, las personas que ahí trabajan o estudian les dedican gran parte de su tiempo, y algunas gran parte de su vida, por lo que los determinantes de salud están regidos en gran medida por las condiciones o características sociales en las cuales esas personas viven, estudian o trabajan en la universidad.
Por otra parte, las instituciones de educación superior se relacionan y utilizan un amplio grupo de servicios vinculados con la salud, el deporte, el transporte, la alimentación, la seguridad, etc., que resultan fundamentales para el establecimiento de estilos de vida que promuevan la salud (14).

Al retomar la idea de que las universidades pueden llegar a ser "ciudades saludables", cabe afirmar que la modificación del entorno podría incidir considerablemente en el logro de estilos de vida más saludables. Para ello, deben ocurrir cambios cualitativos al interior de cada universidad, en su estructura física, líneas de desarrollo estratégico, currícula y políticas, aunque dichos cambios deben sustentarse en reformas de los sectores de salud y educación que faciliten, enmarquen y den sentido a las modificaciones locales. De esta manera, se lograrían cambios que promuevan la salud y que sean sustentables en el tiempo, centrados en sólidas convicciones y conocimientos, y factibles de lograr para los miembros de la comunidad universitaria de manera equitativa.

Como lo señala la Guía para universidades promotoras de la salud y otras instituciones de educación superior (14), las universidades tienen la capacidad de generar beneficios para sí mismas, para sus miembros y sus familias. Esto podría obtenerse reconociendo el valor agregado que la promoción de la salud tiene en términos de incremento de satisfacción laboral, como factor motivador para el ingreso de mayor número de estudiantes y como elemento relevante en la formación de profesionales íntegros. Al mismo tiempo, la incorporación de estilos de vida saludables podría disminuir el ausentismo de estudiantes, académicos y administrativos, favorecer las relaciones interpersonales armoniosas y la convivencia, mejorando el clima laboral y de estudio, y contribuyendo a la percepción de la calidad de vida durante el estudio y trabajo. Por último, en el largo plazo la promoción de la salud en la universidad favorecerá cambios en la situación de salud del país a través de las generaciones de estudiantes egresados que fomentarán modificaciones para lograr el bienestar y la salud de las instituciones y comunidades en las que trabajen y de las familias que formen.

\section{SYNOPSIS}

\section{Universities and health promotion: how can the two come together?}

Universities play a leading role in the communities in which they are found. Focused on leadership and developing knowledge, universities are the stage on which community members are formed. Through education, research, and by spreading knowledge, universities guide and support changes at the national and international levels. The uni- 
versity that advances health is one that joins health promotion with its purpose, aiming to foster human development and improve quality of life for its students and employees, thus impacting both the labor and social circles. It aspires to take a leading role in cultural change by raising awareness among the more educated, open, enterprising, and upstanding, and those committed to progress.

This article's objective is to share a reflective analysis regarding the relationship between the university and health promotion in order to motivate and recommend action items to other universities advanced in this area. This analysis stems from experience gained over several years at the Pon- tificia Universidad Católica of Chile, through its health program, UC Saludable, in addition to a systematic review of the literature covering several years. The analysis develops four concepts that are central to all universities and that frame the health promotion effort: respond to health issues and educate the public; produce and disseminate health promotion knowledge; direct and support awareness of self-care and healthy lifestyles; and be a model change agent.

Key words: health promotion; education, higher; quality of life; human development; Chile.

\section{REFERENCIAS}

1. Rosso P. Discurso con motivo de la Inauguración del año académico 2000. Un proyecto para el siglo XXI, Rectoría. Centro de Extensión. Pontificia Universidad Católica de Chile. Santiago, Chile. [Sitio en Internet] Hallado en: http:/ / www.uc.cl. Acceso el 21 septiembre 2006.

2. Costa I. Desarrollo y salud: la Declaración de Alma Ata y movimientos posteriores. Rev Latinoam Enfermagem. 2004; 12(3):451-2

3. Ottawa Charter for Health Promotion. Can J Pub Health. 1986;77(6):425-30.

4. Sepúlveda LE. Algunas consideraciones respecto de la estrategia "Ambientes saludables" de la promoción de la salud [publicación periódica en línea] 2005. Hallado en: URL: http://lunazul. ucaldas.edu.co/downloads/202cbbe4 Revista21_4.pdf.

5. Toledo A. Las conferencias internacionales de promoción de la salud realizadas hasta el 2005 [publicación periódica en línea] 2005. Hallado en: URL: http:/ / www.ilustrados.com/publicaciones/ EEFVlZVVZIPiSEaNAe.php

6. El Salvador. Ministerio de Salud Pública y Asistencia Social. Marco normativo cronológico de promoción de la salud en El Salvador. 2002. [publicación periódica en línea] 2005. Hallado en: URL: http:/ / www.paho.org.

7. OPS-OMS. Boletín electrónico. 1(4) [publicación periódica en línea] 2005. Hallado en: URL: http:/ / paho.org.

8. McElligott, J. Carta de Bangkok para la Promoción de la Salud en un Mundo Globalizado. Noncommunicable Diseases and Mental Health World Health Organization. 2005. [publicación periódica en línea] 2005. Hallado en: URL: http:/ / www.comminit.com/en/node/ 195838.

9. Organización Mundial de la Salud. Declaración de Jakarta. Nuevos actores para tiempos nuevos: la promoción de la salud hacia el siglo XXI. Cuarta Conferencia Internacional sobre Promoción de la Salud. Jakarta, Indonesia, 21-25 de julio de 1997. [Sitio en Internet] Hallado en: http://www.who.int Acceso 13 de enereo 2007.
10. III Congreso de las Américas de Municipios y Comunidades Saludables. Declaración de Medellín "Mejor calidad de vida para los habitantes de las Américas en el Siglo XXI". Medellín Colombia [publicación periódica en línea] 1999. Hallado en: URL: http: / / www.who.org

11. División de Promoción y Protección de la Salud. Guías para preparar el informe del país sobre los logros en promoción de la salud con respecto a los compromisos en la Declaración de México. Foro de Promoción de la Salud. [publicación periódica en línea] 2005. Hallado en: URL: http://who.org.

12. BIREME/OPS/OMS. Newsletter BVS 059 01/septiembre/2006 [publicación periódica en línea]. Hallado en: URL: http:// newsletter.bireme.br/new/index.php? lang=es\&newsletter=20060901.

13. Organización Panamericana de la Salud Organización Mundial de la Salud. Consejo Directivo $\mathrm{N}^{\circ} 46$, Sesión del Comité Regional $\mathrm{N}^{\circ}$ 57. Washington, USA. [publicación periódica en línea] 2005. Hallado en: URL:http:/ / www.paho.org.

14. Vio F, Lange I. Guía para universidades promotoras de la salud y otras instituciones de educación superior. $1^{\mathrm{a}} \mathrm{ed}$. Santiago: Ministerio de Salud; 2006.

15. Organización Panamericana de la Salud. Promoción de la salud, un enfoque innovador y eficaz. Noticias e información del centenario, información de prensa. [Sitio en Internet] 2002. Hallado en: http:/ / www.paho.org/Spanish/DPI/ 100/100feature47.htm. Acceso el 23 septiembre 2006.

16. Chile. Ministerio de Salud. Encuesta Nacional de Salud. [Sitio en Internet] 2003. Hallado en: http:/ / www.minsal.cl. Acceso el 21 septiembre 2006.

17. Salinas J, Vio F. Promoción de la salud en Chile. Rev Chil Nutr. 2002;29(S1): 164-73.

18. Rosso P. Discurso con motivo de la celebración del Día del Sagrado Corazón, Rectoría. Centro de Extensión. Pontificia Universidad Católica de Chile. [Sitio en Internet] 2006. Hallado en: http:// www.uc.cl. Acceso el 26 septiembre 2006.

19. Urrutia M, Contreras A, Dois A. Modelo de atención de salud basado en el auto- cuidado: inicios y evolución. Revista Horizonte de Enfermería. 2004; 15:81-88.

20. Gil E. Promoción de la salud: Glosario. Subdirección General de Epidemiología, Promoción y Educación para la Salud. OMS: Ginebra. [Sitio en Internet] 1998. Hallado en: http://www.who.int. Acceso el 22 septiembre 2006.

21. Rodríguez J. Psicología social de la salud. $1^{a}$ ed. Madrid: Síntesis; 1995.

22. Comisión Europea. Factores determinantes de la salud. Dirección General Sanidad y Protección de los Consumidores-Salud Pública. [Sitio en Internet]. Hallado en: http: / / ec.europa.eu / health / ph_ determinants/healthdeterminants_es. htm. Acceso el 23 septiembre 2006.

23. Restrepo, H. REDET: el mejor recurso de promoción de la salud [Sitio en Internet] 2003. Hallado en: http://www. rhpeo.org/reviews / 2004/15/index. htm. Acceso el 24 septiembre 2006.

24. Organización Panamericana de la Salud. Área de Desarrollo Sostenible y Salud Ambiental. Unidad de Entornos Saludables. Municipios y ciudades saludables: recomendaciones para la evaluación dirigidas a los responsables de las políticas en las Américas. Washington, USA. [Sitio en Internet]. 2005. Hallado en: http://www.paho.org. Acceso el 23 septiembre 2006.

25. Organización Panamericana de la Salud. (2006). Programa de promoción de la salud en instituciones educativas. [Sitio en Internet]. 2005. Hallado en: http:// www.paho.org. Acceso el 2 diciembre 2006.

26. Organización Mundial de la Salud. Comisión sobre Determinantes Sociales de la Salud. Preguntas y respuestas. [Sitio en Internet]. 2005. Hallado en: http:// www.who.int/social_determinants / strategy/QandAs/es/index.html. Acceso el 23 noviembre 2006.

27. Haughton B. Applying the socialecological model to nutrition issues that promote health and prevent disease. Fam Community Health. 2006 29(1):3-4.

28. Arredondo A, Parada I. Tendencias en la generación y reproducción del conocimiento sobre evaluación económica y salud. Rev Med Ch. 2001;129(8): 925-34. 